\title{
Re-assigning the DFNB33 locus to chromosome 10p11.23-q21.1
}

\author{
Hanen Belguith ${ }^{1,2}$, Saber Masmoudi ${ }^{2}$, Myrna Medlej-Hashim ${ }^{1}$, Eliane Chouery ${ }^{1}$, \\ Dominique Weil ${ }^{3}$, Hammadi Ayadi ${ }^{2}$, Christine Petit ${ }^{3}$ and André Mégarbané ${ }^{*, 1}$
}

${ }^{1}$ Unité de Génétique Médicale, Faculté de Médecine, Université Saint Joseph, Beyrouth, Liban; ${ }^{2}$ Unité Cibles pour le Diagnostic et la Thérapie, Centre de Biotechnologie de Sfax, Tunisie; ${ }^{3}$ Unité de Génétique et Physiologie de l'Audition, INSERM UMRS 587, Institut Pasteur, Paris, France

Homozygosity mapping is a powerful resource for mapping and identifying loci and genes responsible for autosomal recessive disorders. Nevertheless, it could result in the identification of several homozygous regions unrelated to the disease locus or non-informative regions. Previously, a genome-wide screen in a large consanguineous Jordanian family allowed us to assign the DFNB33 locus to chromosome 9q34.3. Sequencing of 23 candidate genes showed 11 SNPs in a heterozygous state in affected individuals. These results ruled out the candidate region on chromosome 9. Using additional markers, we were able to restrict the disease locus to an approximately $14 \mathrm{cM}$ region at chromosome 10, located between markers D10S193 and D10S1784. A maximum LOD score of 3.99 was obtained with two markers, D10S199 and D10S220. The screening of two candidate genes, $C X 40.1$ and $F X Y D 4$, failed to reveal any disease-causing mutations. European Journal of Human Genetics (2009) 17, 122-124; doi:10.1038/ejhg.2008.155; published online 10 September 2008

Keywords: hearing impairment; locus, DFNB33, homozygosity mapping; candidate gene

\section{Introduction}

Up to the present, at least 52 loci responsible for autosomal recessive non-syndromic hearing impairment (ARNSHI) have been published and 26 genes were identified (Hereditary Hearing Loss Homepage: http://webh01.ua. ac.be/hhh/). Most of the findings were made by studying consanguineous families using the identity by descent. However, this strategy faces two major problems: the presence of partially or totally non-informative markers and the identification of several homozygous regions unrelated to the disease locus that may lead to a mis-localization.

In this study, we reassign the DFNB33 locus, previously mapped on chromosome $9 \mathrm{q} 34.3,{ }^{1}$ to chromosome

*Correspondence: Dr A Mégarbané, Medical Genetics Unit, Universite Saint Joseph, 42, rue de Grenelle, Paris 75007, France.

Tel: + 339611421 252; Fax: + 339611421023 ;

E-mail: megarbane@usj.edu.lb

Received 13 March 2008; revised 9 July 2008; accepted 25 July 2008; published online 10 September 2008 10p11.23-q21.1. Two candidate genes, $C X 40.1$ and FXYD4, were sequenced but found not responsible for the disease.

Materials and methods

Family data

The Jordanian consanguineous family under study has been described earlier. ${ }^{1}$ Briefly, four patients present a prelingual ARNSHI.

Genotyping and linkage analysis

A genome-wide screen was initially carried out for this family in 2002. ${ }^{1}$ Additional fluorescent dye-labelled microsatellite markers were genotyped using an $\mathrm{ABI}$ PRISM 3100-Avant automated DNA sequencer (Applied Biosystems, USA). We used the True Allele PCR Premix (Applied Biosystems, USA) according to the manufacturer's instructions.

Two-point LOD scores were calculated using the MLINK software from the Linkage package v5.1. ${ }^{2}$ Hearing 
impairment in the family was analysed as an autosomal recessive trait with full penetrance. An estimated frequency of $10^{-4}$ was used for the disease-causing allele. Meiotic recombination frequencies were assumed to be equal for males and females. The polymorphic markers' allele frequencies were assumed to be equal.

\section{Candidate genes analysis}

Twenty-five genes were screened for the presence of genetic variants by direct fluorescent sequencing. Primers were designed in the introns using primer3 software (http://frodo.wi.mit.edu/cgi-bin/primer3/primer3_www.cgi). The resulting PCR products were fluorescently sequenced using the ABI PRISM BigDye terminator cycle sequencing reaction kit and loaded on the automated sequencer (Applied Biosystems).

\section{Results}

Sequencing of 23 positional candidate genes at the originally localized DFNB33 locus on chromosome 9q34.3 showed 11 single nucleotide polymorphisms (SNPs) in the heterozygous state in affected individuals (Figure 1) allowing this locus to be ruled out as the true disease-related region.

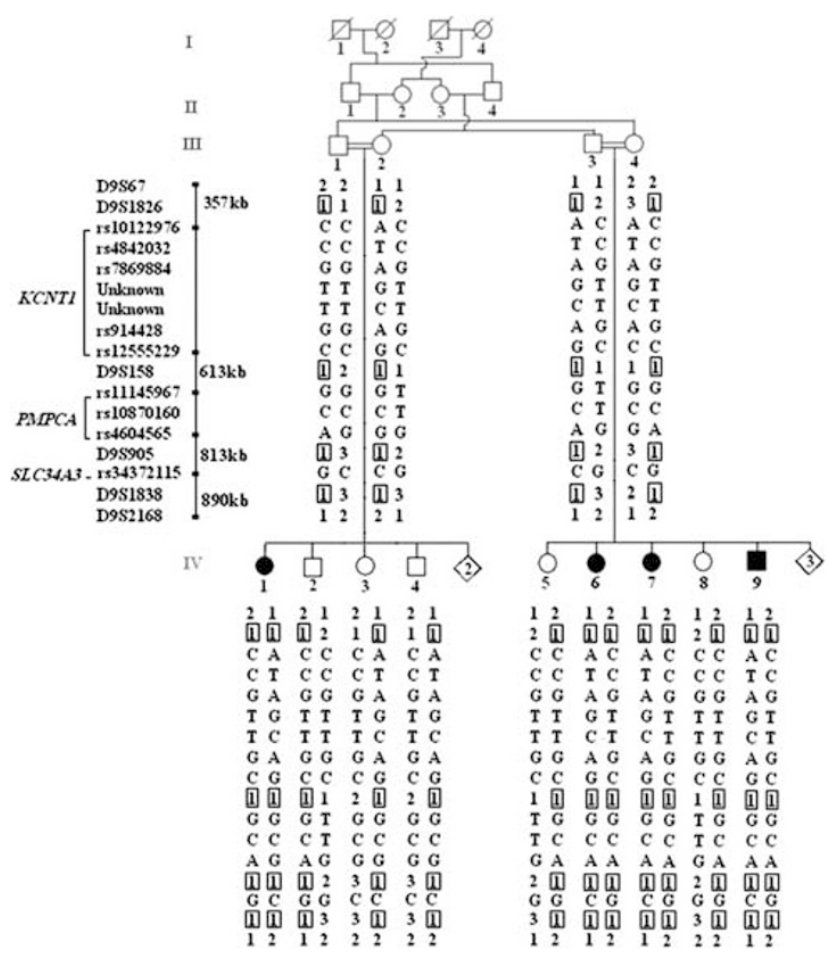

Figure 1 Haplotype reconstruction for 6 microsatellite markers and 11 SNPs at chromosomal region 9q34.3. Black symbols represent individuals affected by hearing impairment. Clear symbols represent unaffected individuals.
Reanalysis of the results of the genome-wide screen showed another semi- informative region on chromosome 10. Screening of 11 additional microsatellite markers allowed us to find evidence of linkage at markers D10S208, D10S199, D10S1791, D10S196 and D10S220. Linkage was confirmed by two-point LOD scores with $Z_{\max }=3.99$ at $\theta=0.00$ for markers D10S199 and D10S220 on chromosome 10p11.23-q21.1 (Figure 2). The homozygous candidate interval, flanked by markers D10S193 and D10S1784, spans an interval of $24 \mathrm{Mb}$ (http://www. genome.ucsc.edu, May 2006 assembly), which corresponds to $13.8 \mathrm{cM}$ (http://research.marshfieldclinic.org/genetics). Sequencing of two candidate genes, CX40.1 and FXYD4, did not reveal any potentially causative variants.

\section{Discussion}

In a previous study, the DFNB33 locus was assigned to the telomeric side of chromosome 9q34.3. ${ }^{1}$ The absence of pathogenic variants in 23 candidate genes and the presence of 11 SNPs in the heterozygous state in affected individuals prompted us to conclude that the region published on chromosome 9q34.3 was not truly because of identity by descent but was rather homozygous by

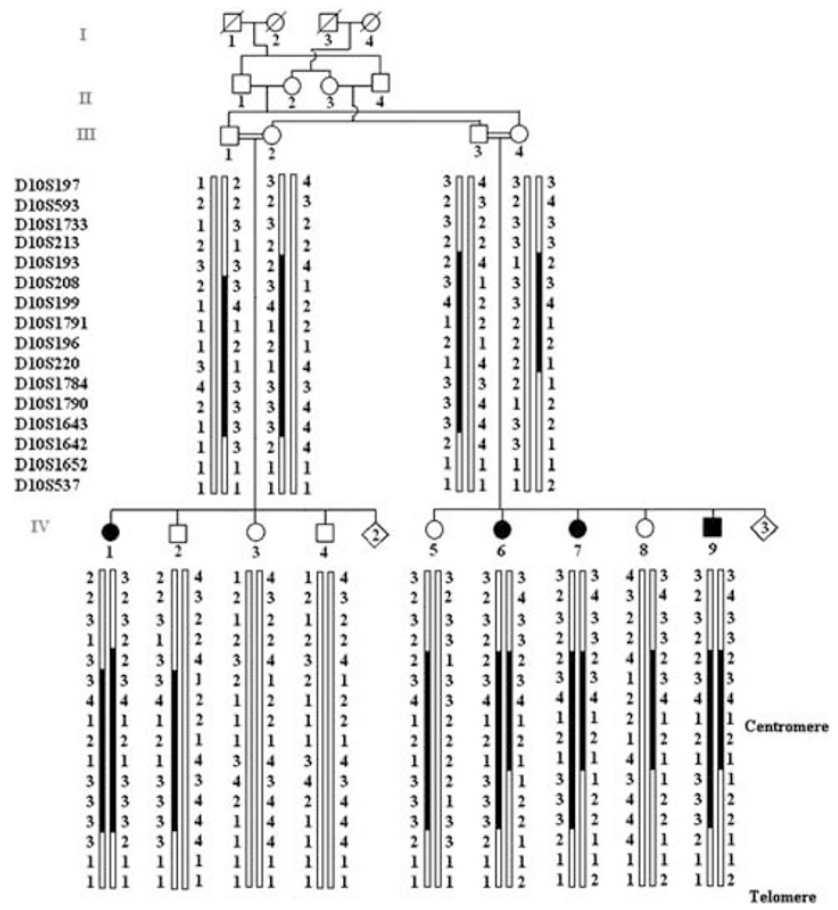

Figure 2 Haplotype reconstruction at chromosome 10p11.23-q21.1 for a Jordanian family. The chromosome assumed to be carrying the disease allele is indicated by a black bar. The critical interval lies within the $13.8 \mathrm{cM}$ region delimited by D10S193 $(59.03 \mathrm{cM})$ and D10S1784 $(72.90 \mathrm{cM})$ markers (http://research.marshfieldclinic. org/genetics) 
chance. Further work-up showed the linkage to chromosome 10p11.23-q21.1.

Four other loci and three genes for inherited ARNSHI have been mapped earlier on chromosome 10 (Hereditary hearing loss homepage: http://webhost.ua.ac.be/hhh/), but none of them belong to the interval assigned to DFNB33.

Owing to the limited number of meioses, the genetic region for the DFNB33 locus is large with more than 75 genes, making identification of the causative one difficult. Moreover, this region is duplicated, complicating the search for causative mutation. Among the known genes, CX40.1 belongs to a family of connexin proteins. Several different connexin sub-units were reported to be expressed in the mammalian inner ear. ${ }^{3}$ Mutations in these genes were responsible for various types of ARNSHI (Hereditary hearing loss homepage: http://webhost.ua.ac.be/hhh/). The second candidate gene screened was FXYD4. ${ }^{4}$ FXYD4, originally named CHIF for channel-inducing factor, is expressed in the mouse cochlea. ${ }^{5}$ It modulates the voltage sensitivity of the potassium channel, KCNQ1. ${ }^{6}$ This channel is expressed in the cochlea and is involved in the transport of salt and water. ${ }^{7}$ Mutations in KCNQ1 have been shown to cause the Jervell and Lange-Nielsen syndrome with congenital deafness, prolonged QT interval and sudden death. ${ }^{8}$ Sequencing of the Cx40.1 and FXYD4 candidate genes showed no pathogenic variations.

In conclusion, we have reassigned the DFNB33 locus to chromosome 10p11.23-q21.1. Our results confirmed the limitation of the use of identity by descent in mapping genes. Semi-informative or non-informative regions should not be discarded, and the absence of informative microsatellite markers can be contoured by the investigation of SNPs alleles.

\section{Acknowledgements}

We are thankful to Dr Hela Azaiez for a critical reading of the paper. In Tunisia, this work was supported by 'Ministère de l'Enseignement Supérieur, de la Recherche Scientifique et de la Technologie' Tunisia and the European Commission FP6 Integrated Project EUROHEAR, LSHG-CT-20054-512063.

\section{References}

1 Medlej-Hashim M, Mustapha M, Chouery E et al: Non syndromic recessive deafness in Jordan: mapping of a new locus to chromosome 9q34.3 and prevalence of DFNB1 mutations. Eur J Hum Genet 2002; 10: 391-394.

2 Lathrop GM, Lalouel JM: Easy calculations of lod scores and genetic risks on small computers. Am J Hum Genet 1984; 36: 460-465.

3 Zhao HB, Kikuchi T, Ngezahayo A, White TW: Gap junctions and cochlear homeostasis. J Membr Biol 2006; 209: 177-186.

4 Sweadner KJ, Rael E: The FXYD gene family of small ion transport regulators or channels: cDNA sequence, protein signature sequence and expression. Genomics 2000; 68: 41-56.

5 Sajan SA, Warchol ME, Lovett M: Toward a systems biology of mouse inner ear organogenesis: gene expression pathways, patterns and network analysis. Genetics 2007; 177: 631-653.

6 Jespersen T, Grunnet M, Rasmussen $\mathrm{HB}$ et al: The corticosteroid hormone induced factor: a new modulator of KCNQ1 channels? Bioch and Bioph Res Comm 2006; 341: 979-988.

7 Warth R, Barhanin J: The multifaceted phenotype of the knockout mouse for the KCNE1 potassium channel gene. Am J Physiol Regul Integr Comp Physiol 2002; 282: 639-648.

8 Neyroud N, Tesson F, Denjoy I et al: Anovel mutation in the potassium channel gene KVLQT1 causes the Jervell and LangeNielsen cardioauditory syndrome. Nat Genet 1997; 15: 186-189. 